\title{
Turmeric Extract May Improve Irritable Bowel Syndrome Symptomology in Otherwise Healthy Adults: A Pilot Study
}

\author{
RAFE BUNDY, Ph.D., ${ }^{1}$ ANN F. WALKER, Ph.D., RICHARD W. MIDDLETON, Ph.D., ${ }^{2}$ \\ AND JONATHAN BOOTH, M.D. ${ }^{3}$
}

\begin{abstract}
Objectives: To assess the effects of turmeric (Curcuma longa) extract on irritable bowel syndrome (IBS) symptomology in otherwise healthy adults.

Design: Partially blinded, randomized, two-dose, pilot study.

Subjects: Five hundred (500) volunteers were screened for IBS using the Rome II criteria. Two hundred and seven (207) suitable volunteers were randomized.

Interventions: One or two tablets of a standardized turmeric extract taken daily for 8 weeks.

Outcomes measures: IBS prevalence, symptom-related quality of life (IBSQOL) and self-reported effectiveness.

Results: IBS prevalence decreased significantly in both groups between screening and baseline (41\% and 57\%), with a further significant drop of 53\% and $60 \%$ between baseline and after treatment, in the one- and two-tablet groups respectively $(p<0.001)$. A post-study analysis revealed abdominal pain/discomfort score reduced significantly by $22 \%$ and $25 \%$ in the one- and two-tablet group respectively, the difference tending toward significance $(p=0.071)$. There were significant improvements in all bar one of the IBSQOL scales of between 5\% and $36 \%$ in both groups, approximately two thirds of all subjects reported an improvement in symptoms after treatment, and there was a favorable shift in self-reported bowel pattern. There were no significant differences between groups.

Conclusions: Turmeric may help reduce IBS symptomology. Placebo controlled trials are now warranted to confirm these findings.
\end{abstract}

\section{INTRODUCTION}

$\mathbf{I}^{\mathrm{r}}$ rritable bowel syndrome (IBS) is the most common disorder seen in gastroenterology practices (Olden, 2002). It is a functional disorder characterized by symptoms including abdominal pain, altered bowel habits, and changes in stool frequency (Camilleri, 2001). There is also evidence that health-related quality of life is significantly reduced in patients suffering from IBS compared to healthy controls (El-Serag et al., 2002).

The causes of IBS are not clear. Proposed hypotheses include infection and antibiotic use, both of which may lead to changes in colonic microflora. It has been recently suggested that a low-grade inflammation of the gut mucosa may underlie some symptomology (Barbara et al., 2002).

There is no accepted cure for IBS. Phytochemicals in the form of herbal remedies have offered alternative treatments, for example, peppermint oil (Pittler and Ernst, 1998). Artichoke leaf extract (ALE) has also been shown to reduce symptom severity in patients with dyspepsia identified as suffering from IBS (Bundy et al., 2004; Walker et al., 2001).

Turmeric (Curcuma longa) has been traditionally used in Indian, Chinese, and Western herbal medicine for conditions such as poor digestion, abdominal pain, and distension.

\footnotetext{
${ }^{1}$ Hugh Sinclair Unit of Human Nutrition, The University of Reading, Reading, UK.

${ }^{2}$ Lichtwer Pharma (UK) Ltd., Marlow, UK.

${ }^{3}$ Gastroenterology Department, The Royal Berkshire Hospital, Reading, UK.
} 
Turmeric and its extracts, namely curcumin, have been shown to possess anti-inflammatory, choleretic, antimicrobial, and carminative actions, which would be useful in disturbed gastrointestinal (GI) tract function (Mills and Bone, 2000). Recent evidence has shown that turmeric inhibits the expression of both cyclooxygenase- 2 and inducible nitric oxide synthetase (both of which mediate inflammatory processes) through a common molecular pathway (Surh et al., 2001).

The objective of the present pilot study was to assess the effects of turmeric supplementation on IBS prevalence, symptom-related QoL, and self-reported effectiveness in otherwise healthy adults suffering IBS.

\section{MATERIALS AND METHODS}

\section{Volunteers}

Five hundred (500) potential volunteers who had self-reported IBS symptoms for at least 3 months were recruited through newspaper and magazine articles in the United Kingdom. Respondents excluded from the trial included: those who did not meet the current Rome II criteria for IBS (based on the simplified modular format); those currently suffering major organ pathology, gallstones, chronic fatigue syndrome; those taking anticoagulant or antiplatelet medicine; and pregnant and lactating women. All subjects gave informed consent prior to participation and understood that they could withdraw at any time for any reason. The study was approved by the University of Reading Ethics and Research Committee (Reading, UK) and the West Berkshire Local Research Ethics Committee.

\section{Study design}

The design of the study was partially blinded, randomized, two-dose, with outcomes assessed through postal questionnaires. Those suitable for study were randomized using MINIM software (London Hospital Medical College, UK) with minimization for gender, age, and IBS type into two groups, to receive either $72 \mathrm{mg}$ (1 tablet) or $144 \mathrm{mg}$ (2 tablets) of a standardized turmeric extract daily (Cynara ${ }^{\mathrm{TM}}$ Turmeric, Lichtwer Pharma (UK) Ltd., Marlow, UK) for 8 weeks. A third party not connected with the trial coded treatment groups. Volunteers were aware that there were two groups but they were not told which group they were in.

Tablets were mailed to volunteers with instructions on how to take the medication (one or two tablets each day with breakfast) and copies of two outcome questionnaires. Further questionnaires were sent at the end of the trial. Questionnaires were returned by mail.

The primary outcome questionnaire was based on the ROME II criteria for IBS in the comprehensive integrative format (Drossman, 1999). A secondary outcome was the IBS Quality of Life (QoL) questionnaire (Hahn et al., 1997). A further questionnaire allowed volunteers to self-report the perceived benefit of the intervention on symptoms and describe any side-effects.

\section{Statistical analysis}

Sample size was calculated as the numbers needed to detect a significant difference between two groups based on a decrease in IBS prevalence of $25 \%$ with one tablet and $50 \%$ with two tablets $(\alpha=0.05$ and $\beta=0.80)$, allowing for a $25 \%$ dropout rate. This was based on other studies conducted within the research group with artichoke leaf extract (Bundy et al., 2004; Walker et al., 2001) because there were no preexisting data for turmeric and IBS. Data were analyzed on an intention-to-treat (ITT) basis. Mann-Whitney $U$ tests were used to test for differences in independent samples and Wilcoxon signed ranks tests were use to test for differences between related samples, using SPSS 11.0 for Windows (SPSS Inc., Chicago, IL).

\section{RESULTS}

\section{Volunteers}

Two hundred and seven (207) volunteers were randomized. The flow of volunteers through each stage of the trial is detailed in Figure 1, as based on Consolidated Standards of Reporting Trials (CONSORT) recommendations (Moher et al., 2001). More than $70 \%$ of volunteers were female, the majority were over 50 years old, and mean body-mass index was 26 in both groups.

\section{Primary outcome}

IBS prevalence at baseline was significantly lower than at screening, with a $41 \%(n=39)$ and $57 \%(n=56)$ reduction in the one- and two-tablet groups, respectively $(p<$ $0.001)$. After treatment with turmeric extract, there were further significant falls in IBS prevalence of $53 \%$ in the onetablet and $60 \%$ in the two-tablet groups ( $p<0.001$; Fig. 2 ), this difference not being significant between groups after adjusting for baseline values.

The unexpected decrease in IBS incidence from screening to baseline meant the power of the study to reveal a difference between the two groups was reduced. Therefore, an unplanned post-study analysis of abdominal pain/discomfort scores (one of the defining ROME II criteria for IBS) was performed on the entire ITT group. Significant reductions in score of $22 \%$ and $25 \%$ were noted in the one-tablet and the two-tablet groups respectively ( $p<0.001)$, and the difference between groups tended toward significance $(p=0.071)$.

\section{Secondary outcomes}

Baseline IBSQOL values were similar for both groups. After treatment, there were significant improvements in all except one of the domain scores of between $5 \%$ and $36 \%$. 


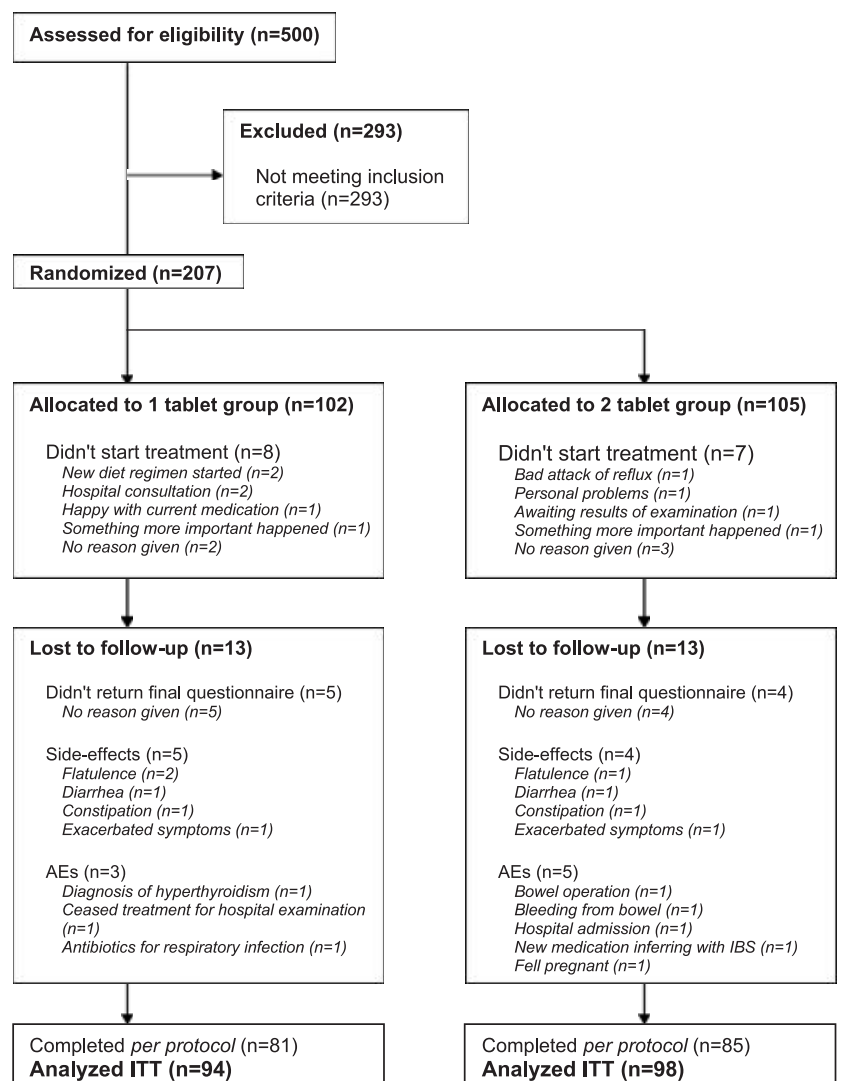

FIG. 1. Flow of volunteers through the study based on Consolidated Standards of Reporting Trials recommendations. AEs, adverse events; ITT, intention-to-treat.

Sixty-seven percent $(67 \%)$ and $70 \%$ of volunteers in the one- and two-tablet groups, respectively, self-reported a definite or some improvement in their IBS after treatment. Only one person in each group reported a worsening of symptoms.

Only $9 \%$ and $10 \%$ of volunteers reported a normal bowel pattern at baseline, which significantly increased to $29 \%$ and $32 \%$ after treatment in the one- and two-tablet groups respectively $(p<0.001)$.

There were no significant differences between groups in any of the secondary outcomes.

\section{Side-effects}

No major side-effects were reported. Prevalent minor side-effects included flatulence, which affected approximately one quarter of all subjects, and dry mouth. Side-effects led to the withdrawal of nine subjects (Fig. 1).

\section{DISCUSSION}

The present pilot trial studied the effect of a standardized turmeric extract on symptoms of IBS in otherwise healthy adults. Compliance rate in the study was good, and we feel that providing volunteers with a clear explanation of the study rationale and making them feel valued as participants played a part in this.

A number of possible reasons are proposed for the unexpected decrease in IBS prevalence between screening and baseline.

First, IBS symptoms may have spontaneously improved over the average 3-month period between screening and baseline (Talley et al., 1992). Second, the screening questionnaire was completed approximately 1 month before Christmas, an often stressful period that may have exacerbated symptoms. Third, the anticipation of being included onto the trial may have brought improvement in some people.

There are also concerns over variations in the two versions of the ROME II questionnaires used at screening and baseline. It appears that the validity of the modular format of the questionnaire used during screening remains to be established, and while the integrative format used at baseline and final is based on a valid tool, it too has not undergone rigorous testing (N.J. Talley, personal communication).

The decrease in IBS prevalence between screening and baseline produced an unbalanced data set and a corresponding drop in statistical power. Measuring the change in abdominal pain/discomfort score in the full ITT dataset allowed us to increase statistical power and examine any changes using balanced group numbers. The finding of a tendency toward a significant improvement in score in the two-tablet compared to the one-tablet group is promising.

Baseline values obtained from IBSQOL questionnaire were similar in both groups to those reported previously (Hahn et al., 1997). The significant improvement in both groups in the present study is encouraging.

Self-reported effectiveness of treatment was positive in both groups with respect to improvements in IBS symptomology and usual bowel pattern. There is little doubt, though, that the placebo effect contributed to the improvements seen in the present study. However, it has been stated that one of the most important aspects of treatment of IBS

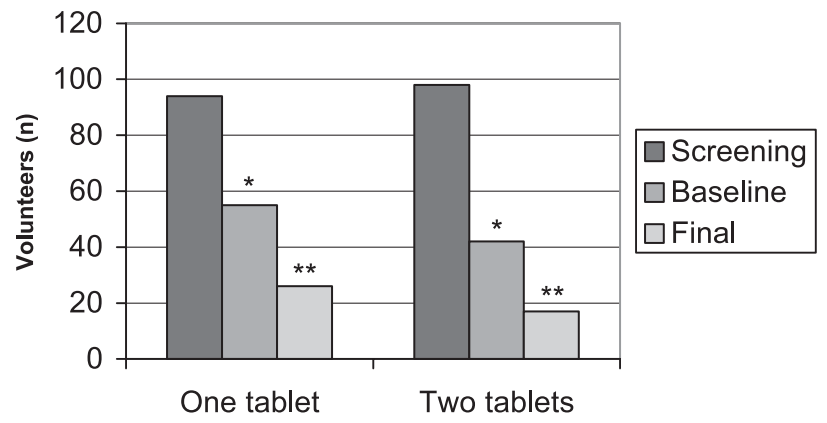

${ }^{*} P<0.001$ Screening to Baseline

FIG. 2. Prevalence of irritable bowel syndrome (IBS) at screening, baseline, and after 8 weeks of treatment with turmeric (Circuma longa) extract, in two doses, given to volunteers who have IBS. 
is to establish a good and lengthy physician-patient relationship, which has been deemed to be "as vital as any medication" (Maxwell et al., 1997). At no point in the current study was there personal contact between investigators and volunteers, in the hope of minimizing this effect.

It is also worth reiterating that IBS has a complex aetiology, and as such it is unlikely that a single intervention will have a universal effect (i.e., "one size does not fit all"). It was clear in that in a number of cases the effects of turmeric intervention had been beneficial, to the point where one subject in the two-tablet group reported a vast improvement after 9 years of being "a prisoner in my own home because I could not be too far from a toilet. . . ." As with any complex illness, there will be responders and nonresponders to a particular treatment.

This pilot study has produced promising results, suggesting the implementation of a placebo-controlled clinical trial to investigate turmeric extract further in treating IBS symptomology.

\section{ACKNOWLEDGMENTS}

We are grateful to the volunteers who participated in this study and to Lichtwer Pharma (UK) Ltd. for the donation of the turmeric extract (Cynara ${ }^{\mathrm{TM}}$ Turmeric) and for funding Dr. Bundy's fellowship. We are also grateful to Mrs. Ruba Carroll, Mrs. Jan Luff, and Miss Jessica Hope for their assistance.

\section{REFERENCES}

Barbara G, De Giorgio R, Stanghellini V, Cremon C, Corinaldesi $\mathrm{R}$. A role for inflammation in irritable bowel syndrome? Gut 2002;51(Suppl 1):I41-I44.

Bundy R, Walker AF, Middleton RW, Marakis G, Booth JCL. Artichoke leaf extract improves symptoms of irritable bowel syndrome and quality of life in otherwise healthy volunteers suffering concomitant dyspepsia: A subset analysis. J Altern Complement Med 2004;10:667-669.
Camilleri M. Dyspepsia, irritable bowel syndrome, and constipation: Review and what's new. Rev Gastroenterol Disord 2001;1:2-17.

Drossman DA, ed. ROME II: The Functional Gastrointestinal Disorders, 2nd ed. McLean, VA: Degnon Associates Inc, 1999.

El-Serag HB, Olden K, Bjorkman D. Health-related quality of life among persons with irritable bowel syndrome: A systematic review. Aliment Pharmacol Ther 2002;16:1171-1185.

Hahn BA, Kirchdoerfer LJ, Fullerton S, Mayer E. Evaluation of a new quality of life questionnaire for patients with irritable bowel syndrome. Aliment Pharmacol Ther 1997;11:547-552.

Maxwell PR, Mendall MA, Kumar D. Irritable bowel syndrome. Lancet 1997;350:1691-1695.

Mills S, Bone K. Principles and Practice of Phytotherapy. London: Churchill Livingstone, 2000

Moher D, Schulz KF, Altman DG. The CONSORT statement: Revised recommendations for improving the quality of reports of parallel-group randomised trials. Lancet 2001;357:1191-1194.

Olden KW. Diagnosis of irritable bowel syndrome. Gastroenterology 2002;122:1701-1714.

Pittler MH, Ernst E. Peppermint oil for irritable bowel syndrome: A critical review and metaanalysis. Am J Gastroenterol 1998;93:1131-1135.

Surh YJ, Chun KS, Cha HH, Han SS, Keum YS, Park KK, Lee SS. Molecular mechanisms underlying chemopreventive activities of anti-inflammatory phytochemicals: Down-regulation of COX-2 and iNOS through suppression of NF-kappa B activation. Mutat Res 2001;480-481:243-268.

Talley NJ, Weaver AL, Zinsmeister AR, Melton LJ, 3rd. Onset and disappearance of gastrointestinal symptoms and functional gastrointestinal disorders. Am J Epidemiol 1992;136:165-177.

Walker AF, Middleton RW, Petrowicz O. Artichoke leaf extract reduces symptoms of irritable bowel syndrome in a post-marketing surveillance study. Phytother Res 2001;15:58-61.

Address reprint requests to: Rafe Bundy, Ph.D.

Hugh Sinclair Unit of Human Nutrition

School of Food Biosciences

University of Reading

Reading, United Kingdom

E-mail: r.bundy@reading.ac.uk 